\title{
Long non-coding RNA LINC01018 inhibits the progression of acute myeloid leukemia by targeting miR-499a-5p to regulate PDCD4
}

\author{
HONG ZHOU $^{1}$, PENGFEI SHI ${ }^{1}$, XIAOFENG JIA ${ }^{2}$ and QIANFU XUE ${ }^{3}$ \\ ${ }^{1}$ Department of Hematology, Affiliated Hangzhou First People's Hospital, Zhejiang University School of Medicine, \\ Hangzhou, Zhejiang 310006; ${ }^{2}$ College of Life Sciences, China Jiliang University, Hangzhou, Zhejiang 310018; \\ ${ }^{3}$ Department of Hematology, Yong Chuan Hospital of Chongqing Medical University, Chongqing 402160, P.R. China
}

Received June 30, 2020; Accepted October 29, 2020

DOI: $10.3892 / \mathrm{ol} .2021 .12802$

\begin{abstract}
Acute myeloid leukemia (AML) is a highly heterogeneous disease with a very high mortality rate. In recent years, an increasing number of studies have proven that long non-coding RNAs (lncRNAs) and microRNAs (miRNAs) may serve as useful biomarkers in various cancer types. However, the mechanism of LINC01018 and miR-499a-5p in AML requires further investigation. The mRNA expression of LINC01018, miR-499a-5p and PDCD4 in AML tissues and cells was detected using reverse transcription-quantitative polymerase chain reaction. Cell proliferation was measured using Cell Counting kit-8 and EdU assays. Cell apoptosis was monitored via a TUNEL staining assay. Protein expression of PDCD4, Bax and Bcl-2 was measured using western blot analysis. The interaction between PDCD4 and LINC01018 or miR-499a-5p was verified by RNA pull-down, RIP and dual-luciferase reporter assays. LINC01018 and PDCD4 were downregulated in AML, while miR-499a-5p was upregulated. LINC01018-overexpression suppressed AML cell proliferation and induced AML cell apoptosis, while miR-499a-5p transfection reversed these effects. LINC01018 acted as a sponge of miR-499a-5p, and PDCD4 was demonstrated to be targeted by miR-499a-5p. Knockdown of miR-499a-5p suppressed AML cell proliferation and promoted AML cell apoptosis, but silencing PDCD4 abolished this effect. LINC01018 inhibited AML cell growth by modulating PDCD4 through suppression of miR-499a-5p, providing a feasible theoretical basis for the treatment of AML.
\end{abstract}

Correspondence to: Dr Qianfu Xue, Department of Hematology, Yong Chuan Hospital of Chongqing Medical University, 439 Xuanhua Road, Yongchuan, Chongqing 402160, P.R. China E-mail: xueqf700438@163.com

Key words: LINC01018, microRNA-499a-5p, PDCD4, acute myeloid leukemia, tumor suppressor

\section{Introduction}

Acute myeloid leukemia (AML) is a highly heterogeneous disease that originates from myeloid progenitor cells (1). AML has a very high mortality rate, particularly in patients over 65 years of age (2). At present, although approaches such as chemotherapy and stem cell transplantation have achieved significant success in the treatment of AML, $>70 \%$ of 65-year-old or older patients will die within 1 year of diagnosis $(3,4)$. Even patients who achieve complete remission may eventually die from the emergence of drug resistance, which is a major obstacle for AML treatment (5). Therefore, it is necessary to investigate the molecular mechanisms of AML pathogenesis to develop novel therapeutic strategies.

A total of $90 \%$ of the human genome is comprised of non-coding RNAs (ncRNAs), which are divided into numerous different subtypes (6). As subtypes of ncRNAs, long non-coding RNAs (IncRNAs) and microRNAs (miRNAs) have attracted increasing attention in recent years in the study of AML pathogenesis (7-9). LncRNAs exceed 200 nucleotides in length. Studies have revealed that IncRNAs serve a critical role in numerous life processes, including gene regulation, cell cycle regulation and cell differentiation regulation $(10,11)$. LncRNAs that are abnormally expressed may act as oncogenes or tumor suppressor genes in numerous types of malignancy (12). For example, IncRNA HOTAIR has been demonstrated to be involved in metastasis and poor prognosis of liver, colorectal and pancreatic cancer types (13-15). The expression level of the lncRNA MALAT1 in lung tumors was three-fold higher than that in healthy controls (16). Furthermore, lncRNA LINC01018 was correlated with the molecular pathways of tumors (17). Another study demonstrated that the expression levels of liver metabolic genes were regulated by LINC01018 (18). At present, research on LINC01018 is scarce, and the role of LINC01018 in AML progression and drug resistance is not completely clear.

MiRNAs, short ncRNAs with a length of 19-25 nt (19), have been proven to be associated with the initiation and progression of AML in previous studies. For example, a study undertaken by Song et al (20) determined that miR-22 was a proto-oncogene and that abnormalities in the miR-22-TET2 axis were common 
in hematopoietic malignancies. In addition, Bousquet et al (21) indicated that miR-125b interferes with the differentiation of primary human $\mathrm{CD}_{3} 4^{+}$cells and inhibits terminal differentiation in leukemic cells. At present, studies have demonstrated that miR-499a-5p is associated with the occurrence and development of lung adenocarcinoma, pancreatic cancer and oral squamous cell carcinoma (22-24). However, whether miR-499a-5p serves a role in the pathogenesis of AML remains undetermined.

Programmed cell death 4 (PDCD4) is a tumor repressor that is usually decreased in various cancer types (25-27). It has been shown to effectively inhibit cancer cell promotion, progression and proliferation (28). A recent study has indicated that PDCD4 may suppress mTORC2 activation, which also serves a significant role in the regulation of cell invasion, migration and metastasis (29). Furthermore, several studies have reported that PDCD4 may inhibit the translation of a series of genes, including p53, Bcl-xl and XIAP, thereby suppressing the occurrence of tumors (30-32). However, the role of PDCD4 in AML pathogenesis has not been extensively investigated.

In the present study, the expression levels of LINC01018, miR-499a-5p and PDCD4 were detected in AML tissues and cell lines, and the interaction between PDCD4 and LINC01018 or miR-499a-5p was analyzed, providing a novel available therapeutic target for the treatment of AML.

\section{Materials and methods}

AML tissues and cell lines. A total of 40 bone marrow specimens were collected from patients with AML (mean age, 62.3 years; age range, 32 to 71 years) at the Affiliated Hangzhou First People's Hospital from May 2016 to Feb 2020. Bone marrow specimens were obtained from 40 healthy volunteers (mean age, 60.3 years; age range, 36 to 72 years) at the Affiliated Hangzhou First People's Hospital from May 2018 to Feb 2020. The present study was approved by the Ethics Committee of Affiliated Hangzhou First People's Hospital (Hangzhou, China; approval no. 146-01). Written informed consent was provided by all patients prior to the study start. The clinical characteristics of the patients are presented in Tables I and II. AML HL-60 and THP-1 cell lines and the normal BM HS cell line were supplied by the American Type Culture Collection. All cell lines were cultured in RPMI-1640 medium (Gibco; Thermo Fisher Scientific, Inc.) containing $10 \%$ fetal bovine serum (Gibco; Thermo Fisher Scientific, Inc.). All cells were cultured in a humidified incubator with $5 \% \mathrm{CO}_{2}$ at $37^{\circ} \mathrm{C}$.

Transfection of oligonucleotides. pcDNA3.1/LINC01018, a miR-499a-5p mimic (5'-UUAAGACUUGCAGUGAUG UUU-3'), miR-499a-5p inhibitor (5'-AAACATCTCTGCAAG TCTTAA-3'), miR mimic control (5'-ACUACUGAGUGA CAGUAGA-3'), miR inhibitor control (5'-CAGUACUUUUGU GUAGUACAA-3'), si-PDCD4 (sense, 5'-GGAGCGGUUUGU AGAAGAA dTdT-3' and antisense, 3'-dTdT CCUCGCCAA ACAUCUUCUU-5'), si-control (sense, 5'-UUCCCUUUG UCAUCCUUUGCCU dTdT-3' and antisense, 3'-dTdT AAG GGAAACAGUAGGAAACGGA-5') and a pcDNA3.1 empty vector were all purchased from Shanghai GenePharma Co., Ltd. HL-60 and THP-1 cells were transfected with pcDNA3.1 (50 ng), pcDNA3.1/LINC01018 (50 ng), miR-499a-5p mimic
(100 nM), mimic control (100 nM), miR-499a-5p inhibitor (100 nM), inhibitor control (100 nM), si-PDCD4 (50 nM), and si-control $(50 \mathrm{nM})$ at $37^{\circ} \mathrm{C}$ for $48 \mathrm{~h}$ using Lipofectamine 3000 reagent (Invitrogen; Thermo Fisher Scientific, Inc.). Cells were collected and used $48 \mathrm{~h}$ later for subsequent experimentation.

Reverse transcription-quantitative polymerase chain reaction (RT-qPCR) assay. Total RNA from AML tissues and cells was extracted using TRIzol reagent (Invitrogen; Thermo Fisher Scientific, Inc.) and its quality was detected via NanoDrop 2000c (Thermo Fisher Scientific, Inc.), according to the manufacturer's protocol. Next, the RNA samples were converted into cDNA using M-MLV reverse transcriptase (Invitrogen; Thermo Fisher Scientific, Inc.). The reverse transcription protocol was $70^{\circ} \mathrm{C}$ for $10 \mathrm{~min}$ followed by $2 \mathrm{~min}$ on ice. RT-qPCR was conducted on an ABI 7900 system with SYBR Green Real-Time PCR master mixes (Thermo Fisher Scientific, Inc.). The thermocycling conditions were as follows: Denaturation at $95^{\circ} \mathrm{C}$ for $10 \mathrm{~min}$ followed by 40 cycles of $95^{\circ} \mathrm{C}$ for $15 \mathrm{sec}$ and $60^{\circ} \mathrm{C}$ for $1 \mathrm{~min}$. GAPDH was used as an internal control for LINC01018 and PDCD4, and U6 small nuclear RNA served as a control for miR-499a-5p. The relative expression was analyzed using the $2^{-\Delta \Delta \mathrm{Cq}}$ method (33). The sequences of all primers are presented in Table III.

Cell viability assessment. The effects of LINC01018 and miR-499a-5p on AML cell viability were evaluated using the Cell Counting kit-8 (CCK-8; Sigma-Aldrich; Merck KGaA) assay. AML cells were seeded onto 96 -well plates $\left(4 \times 10^{3}\right)$ and cultured overnight at $37^{\circ} \mathrm{C}$ followed by transfection of the indicated oligonucleotides. After 12, 24, 48 and $72 \mathrm{~h}$ of transfection, the absorbance of each well was detected at $450 \mathrm{~nm}$ by a spectrophotometer. The results represent the mean of three replicates under the same conditions.

Cell proliferation analysis. AML cell proliferation was analyzed by an EdU assay kit (Guangzhou RiboBio Co., Ltd.), according to the manufacturer's protocols. Cell nuclei were stained with Hoechst 3344 ( $4 \mu \mathrm{g} / \mathrm{ml}$, Invitrogen; Thermo Fisher Scientific, Inc.) at room temperature for $30 \mathrm{~min}$. EdU-positive cells were red.

Cell apoptosis analysis. Apoptosis of AML cells was detected by TUNEL staining using a cell death detection reagent, fluorescein (Roche Diagnostics). Briefly, transfected AML cells $\left(1 \times 10^{6}\right)$ were added into the slides with polylysine. Cells were fixed with $2 \%$ formaldehyde at $4^{\circ} \mathrm{C}$ for $30 \mathrm{~min}$ and permeabilized using $0.2 \%$ Triton-X. After washing three times with PBS, cells were processed with $20 \mu \mathrm{g} / \mathrm{ml}$ Proteinase K (Sigma-Aldrich; Merck $\mathrm{KGaA}$; cat. no. P2308) at room temperature for $20 \mathrm{~min}$. Next, cells were washed with PBS three times followed by a $1 \mathrm{~h}$ incubation of the TUNEL reaction mixture at $37^{\circ} \mathrm{C}$ according to the manufacturer's instructions. Nucleic acids were stained using DAPI (1:500, Sigma-Aldrich, cat. no. D9564) for $15 \mathrm{~min}$ at room temperature. Subsequently, cells were incubated with $50 \mu \mathrm{l}$ DAB solution at room temperature for $20 \mathrm{~min}$, and the nuclei were stained using hematoxylin at room temperature for $3 \mathrm{~min}$. After dehydration and transparency, the signals were scanned from 6 random fields using a confocal microscope (Olympus BX51TRF; Olympus Corporation) (magnification, x100) and the 
Table I. Clinical characteristics of patients with AML $(n=40)$.

\begin{tabular}{|c|c|}
\hline Parameter & AML patients, $n$ \\
\hline \multicolumn{2}{|l|}{ Age, years } \\
\hline$>60$ & 32 \\
\hline$\leq 60$ & 8 \\
\hline \multicolumn{2}{|l|}{ Sex } \\
\hline Male & 22 \\
\hline Female & 18 \\
\hline \multicolumn{2}{|l|}{ Diabetes } \\
\hline No & 34 \\
\hline Yes & 6 \\
\hline \multicolumn{2}{|c|}{ Length of hospital stay, days } \\
\hline$>20$ & 11 \\
\hline$\leq 20$ & 29 \\
\hline \multicolumn{2}{|l|}{ Glucocorticoid } \\
\hline No & 40 \\
\hline Yes & 0 \\
\hline \multicolumn{2}{|l|}{ Chemotherapy } \\
\hline No & 40 \\
\hline Yes & 0 \\
\hline \multicolumn{2}{|l|}{ Radiotherapy } \\
\hline No & 40 \\
\hline Yes & 0 \\
\hline \multicolumn{2}{|c|}{ FAB classification } \\
\hline M1-M3 & 17 \\
\hline M4-M6 & 23 \\
\hline \multicolumn{2}{|c|}{ Cytogenetic risk } \\
\hline Favorable & 5 \\
\hline Intermediate & 31 \\
\hline Unfavorable & 4 \\
\hline \multicolumn{2}{|c|}{ NCCN risk stratification } \\
\hline Favorable & 9 \\
\hline Intermediate & 17 \\
\hline Unfavorable & 14 \\
\hline
\end{tabular}

AML, acute myeloid leukemia; FAB, French, American and British; NCCN, National Comprehensive Cancer Network.

number of TUNEL-positive cells was counted. The apoptosis index was used to measure the degree of cell apoptosis.

RNA immunoprecipitation (RIP). RIP was performed to analyze the interplay between LINC01018 and miR-499a-5p using an EZ-Magna RIP kit (Merck KGaA). Following cell lysis, the cell extract was incubated with RIP buffer containing an AGO2 antibody coated on magnetic beads, and an IgG antibody was used as a control. The precipitated RNAs were quantified and purified and then reverse transcribed into cDNA, and RT-qPCR was performed to analyze LINC01018 and miR-499a-5p levels.

Western blotting. Total protein was extracted from HL-60 and THP-1 cells using cell lysis buffer (cat. no. AR0105; Wuhan
Table II. Comparison between patients with AML and controls in age, sex, diabetes and length of stay.

\begin{tabular}{lrrrr}
\hline Parameter & All cases & Health control & AML & P-value \\
\hline Age, years & 32 & 15 & 17 & 0.695 \\
$>60$ & 8 & 5 & 3 & \\
$\leq 60$ & & & & \\
Sex & 22 & 9 & 13 & 0.525 \\
$\quad$ Male & 18 & 10 & 8 & \\
Female & & & & \\
Diabetes & 34 & 19 & 15 & 0.398 \\
$\quad$ No & 6 & 2 & 4 & \\
Yes & & & & \\
Length of hospital & & 5 & 6 & 0.477 \\
stay, days & 11 & 18 & 11 & - \\
$>20$ & 29 & & & \\
$\leq 20$ & & & & \\
\end{tabular}

AML, acute myeloid leukemia.

Boster Biological Technology, Ltd.). Following the protein concentration being determined using a BCA Protein Assay kit (Thermo Fisher Scientific, Inc.), $50 \mu \mathrm{g}$ samples were isolated by $10 \%$ SDS-PAGE gels, transferred onto PVDF membranes (Merck $\mathrm{KGaA}$ ) and blocked with 5\% skimmed milk for $1 \mathrm{~h}$ at room temperature. Subsequently, the membranes were incubated with Bax antibody (dilution, 1:1,000; cat. no., ab182733; Abcam) or Bcl-2 antibody (dilution, 1:1,000; cat. no., ab32124; Abcam) at $4^{\circ} \mathrm{C}$ overnight, followed by incubation with the corresponding HRP-conjugated secondary antibodies (1:2,000; cat. nos. ab205719 or ab6721; Abcam) at room temperature for $1 \mathrm{~h}$. Finally, the protein bands were visualized using an enhanced chemiluminescence reagent (Merck KGaA) and GAPDH was used as an internal control.

Bioinformatics analysis. miR-499a-5p information was obtained through miR Base (http://www.mirbase.org/index. shtml). The prediction of LINC01018 and miR-499a-5p was conducted using starbase v2.0 (http://starbase.sysu.edu. $\mathrm{cn})$. The target genes including PDCD4 of miR-499a-5p were predicted using starbase v2.0, TargetScan (http://www. targetscan.org/) and MicroRanda (http://www.microrna.org/).

Dual-luciferase reporter assay. The interaction between miR-499a-5p and LINC01018 or PDCD4 was verified by dual-luciferase assays. In brief, the wild-type (WT) and mutant (MUT) sequences of LINC01018 or PDCD4, which contained miR-499a-5p binding sites, were amplified and inserted into pmirGLO (Promega Corporation). The recombinant luciferase plasmids were named LINC01018-WT, PDCD4-WT1, PDCD4-WT2, PDCD4-WT3, LINC01018-MUT, PDCD4-MUT1, PDCD4-MUT2, PDCD4-MUT3. AML cells were co-transfected with miR-499a-5p mimics or negative control and the indicated recombinant luciferase plasmids $(0.8 \mu \mathrm{g})$ and $0.08 \mu \mathrm{g}$ renilla plasmid (RL-SV40; Promega Corporation) using Lipofectamine $3000^{\circledR}$ reagent (Invitrogen; 
Table III. Primer sequences used for quantitative PCR.

ID Sequence $\left(5^{\prime}-3^{\prime}\right)$

GAPDH
GAPDH
LINC01018
LINC01018
PDCD4
PDCD4
miR-499a-5p
miR-499a-5p
U6
U6

Forward: TGTTCGTCATGGGTGTGAAC

Reverse: ATGGCATGGACTGTGGTCAT

Forward: TGGATTCACATCTGCTGGGT

Reverse: TGGCCAACATTTGTCAAGGG

Forward: CCTGAATTAGCACTGGATACTCCT

Reverse: CTAGCCTGCACACAATCTACAGTT

Forward: ATGTAGCGTGCGACCG

Reverse: CAGGCTGACGCACTCTGTGCT

Forward: CCATCGGAAGCTCGTATACGAAATT

Reverse: GGCCTCTCGAACTTGCGTGTCAG

miR, microRNA

Thermo Fisher Scientific, Inc.). After $48 \mathrm{~h}$ of transfection, the luciferase activity was analyzed by a dual-luciferase reporter assay system (Promega Corporation) according to the manufacturer's instructions. The results were normalized using Renilla luciferase activity.

Statistical analysis. All results are presented as the mean \pm standard deviation. The two groups were compared using the unpaired Student's t-test, and multiple comparisons were conducted using one-way analysis of variance, followed by Tukey's post hoc test in GraphPad Prism 7 (GraphPad Software, Inc.). Correlation analysis was conducted using the Pearson correlation coefficient. $\mathrm{P}<0.05$ was considered to indicate a statistically significant difference.

\section{Results}

LINC01018 is downregulated while miR-499a-5p is upregulated in AML tissues and cell lines. RNAalifold Web Server was used to obtain the predicted secondary structures of LINC01018: Centroid and minimum free energy predictions. A mountain plot of the secondary structures is shown in Fig. 1A. The expression of LINC01018 and miR-499a-5p in AML tissues and cell lines was measured using RT-qPCR. Compared with that in healthy controls $(n=40)$, the expression level of LINC01018 was decreased, while miR-499a-5p was increased, in AML tissues $(n=40$; $\mathrm{P}<0.05$; Fig. $1 \mathrm{~B}$ and $\mathrm{C})$. The results in Fig. 1D show a negative correlation between LINC01018 and miR-499a-5p expression in patients with AML $(r=-0.3881 ; \mathrm{P}=0.0134)$. In addition, RT-qPCR analysis indicated that LINC01018 levels were markedly decreased in HL-60 and THP-1 cell lines compared with that in HS cells $(\mathrm{P}<0.05$; Fig. $1 \mathrm{E})$. The expression of miR-499a-5p was significantly higher in HL-60 and THP-1 cells than in HS cells $(\mathrm{P}<0.05$, Fig. $1 \mathrm{~F})$. These data demonstrated that LINC01018 was expressed at low levels, while miR-499a-5p was highly expressed, in AML tissues and cell lines.

MiR-499a-5p reverses the suppressive effects of LINC01018-overexpression on AML cell growth. Following investigating the levels of LINC01018 and miR-499a-5p and verifying their correlation, the biological functions of miR-499a-5p and LINC01018 in AML were analyzed. To begin with, RT-qPCR was applied to investigate the overexpression efficiency of LINC01018 in AML cells. The results indicated that the LINC01018 level was significantly higher in the pcDNA3.1/LINC01018 group than in the blank and negative control groups $(\mathrm{P}<0.05$; Fig. 2A). Next, CCK-8 and EdU staining assays were performed to detect the proliferation of HL-60 and THP-1 cells following transfection of LINC01018 alone or LINC01018 plus miR-499a-5p. The results revealed that LINC01018 transfection significantly suppressed cell proliferation; however, this effect was reversed by the overexpression of miR-499a-5p $(\mathrm{P}<0.05$; Fig. 2B-F). Furthermore, the TUNEL assay verified that LINC01018-overexpression increased the apoptosis rate of HL-60 and THP-1 cells, while miR-499a-5p overexpression reversed this effect $(\mathrm{P}<0.05$; Fig. 2G-I). In addition, western blot analysis demonstrated that the protein expression levels of Bax and Bcl-2 were increased in HL-60 and THP-1 cells following transfection with pcDNA3.1/LINC01018, while miR-499a-5p overexpression abolished this upregulation of Bax and Bcl-2 ( $\mathrm{P}<0.05$; Fig. 2J-K). In conclusion, LINC01018-overexpression inhibited the growth of HL-60 and THP-1 cells; however, miR-499a-5p blocked these effects.

LINC01018 acts as a sponge of miR-499a-5p, and PDCD4 is targeted by miR-499a-5p. To further study the mechanism of LINC01018 and miR-499a-5p in AML, the association between LINC01018 and miR-499a-5p and the potential targeted genes of miR-499a-5p was investigated by bioinformatics analysis. To begin with, the transfections were successful with miR-499a-5p or miR-499a-5p inhibitors in all cell types validated by qPCR (Fig. 3A and B). One complementary binding site was identified between LINC01018 and miR-499a-5p (Fig. 3C; upper panel). The luciferase reporter assay results demonstrated that the transfection of miR-499a-5p mimics strongly decreased the luciferase activity of cells following transfection with LINC01018-WT $(\mathrm{P}<0.05$; Fig. 3C; lower panel). Furthermore, three binding sites between 
A

A Predicted secondary structures of

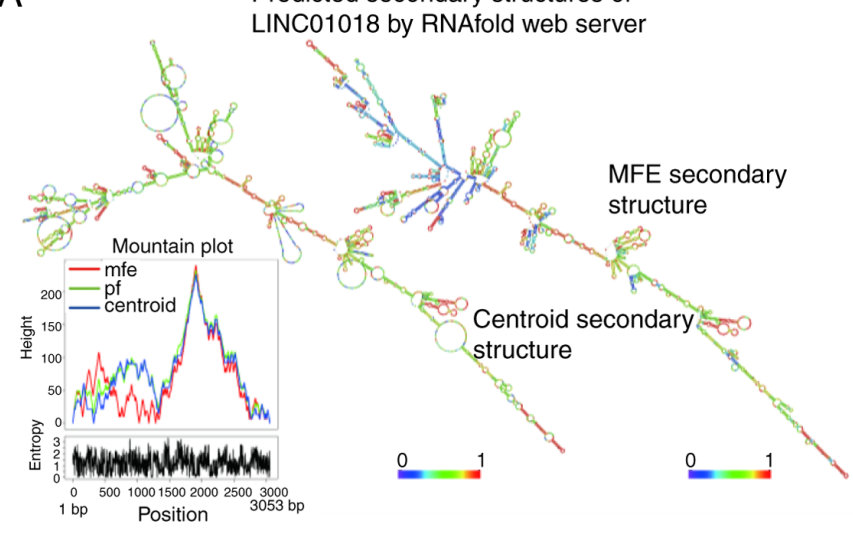

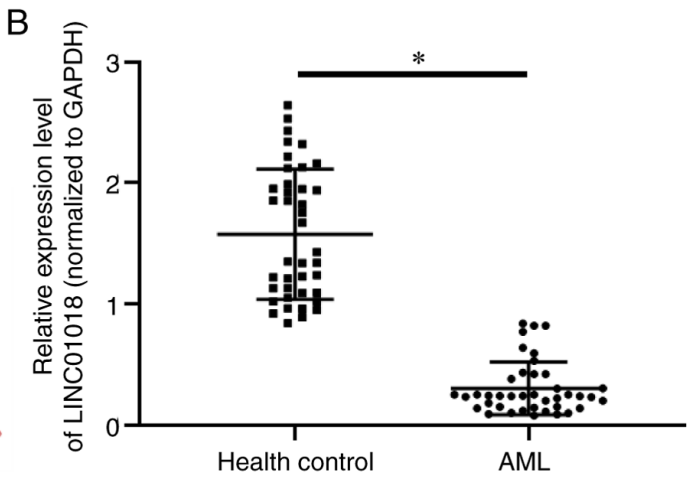

$\mathrm{D}$
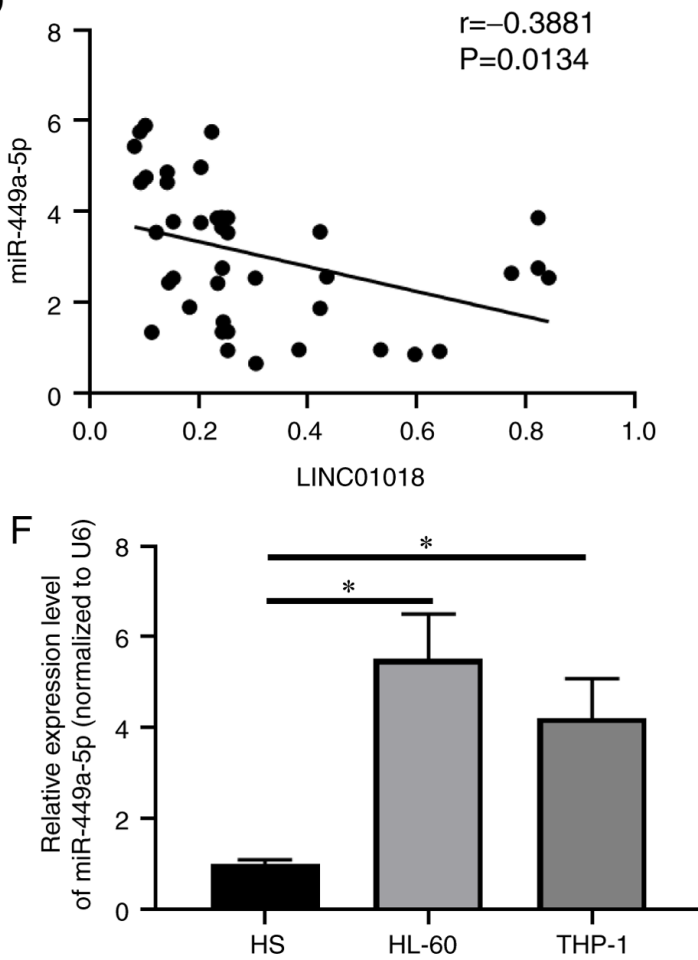

Figure 1. LINC01018 is downregulated, while miR-499a-5p is upregulated in AML tissues and cell lines. (A) The predicted secondary structures of LINC01018, including Centroid and MFE, were obtained using RNAalifold Web Server. (B and C) AML and healthy control tissues were analyzed for LINC01018 and miR-499a-5p expression levels by RT-qPCR. (D) The correlation between the levels of LINC01018 and miR-499a-5p was determined in 40 AML tissues $(\mathrm{r}=-3881 ; \mathrm{P}=0.0134)$. (E and F) AML cell lines were assayed for LINC01018 and miR-499a-5p expression levels using RT-qPCR. HS cells were used as a control. " $\mathrm{P}<0.05$. miR, microRNA; AML, acute myeloid leukemia; RT-qPCR, reverse transcription-quantitative polymerase chain reaction; MFE, minimum free energy.

miR-499a-5p and PDCD4 were predicted using a bioinformatics tool (Fig. 3D; upper panel). The luciferase reporter results demonstrated that the luciferase activities were markedly decreased following co-transfection with miR-499a-5p and PDCD4-WT1, PDCD4-WT2 or PDCD4-WT3 relative to NC mimics, while co-transfection with miR-499a-5p and PDCD4-MUT1, PDCD4-MUT2 or PDCD4-MUT3 caused no notable change relative to $\mathrm{NC}$ mimics $(\mathrm{P}<0.05$; Fig. 3D; lower panel). Next, an RNA pull-down assay was performed to investigate the interaction between LINC01018 and miR-499a-5p. Compared with that in the Bio-probe-NC group, miR-499a-5p was significantly enriched in the Bio-WT-LINC01018 group but not in the Bio-MUT-LINC01018 group ( $\mathrm{P}<0.05$; Fig. 3E). Furthermore, a RIP assay was used to further confirm the interplay between LINC01018 and miR-499a-5p. The results indicated that LINC01018 and miR-499a-5p were significantly enriched in the anti-AGO2 group compared with the anti-IgG group $(\mathrm{P}<0.05$; Fig. 3F). In addition, western blotting was performed to measure PDCD4 protein expression. The expression of PDCD4 was markedly increased in HL-60 and THP-1 cells following knockdown of miR-499a-5p or overexpression of LINC01018 relative to the negative control (Fig. 3G). Taken together, these results indicated that PDCD4 is a target gene of miR-499a-5p and that its expression level is regulated by LINC01018 through miR-499a-5p.

Knockdown of PDCD4 abrogates the effects of the miR-499a-5p inhibitor on AML cell lines. Following confirming the result 


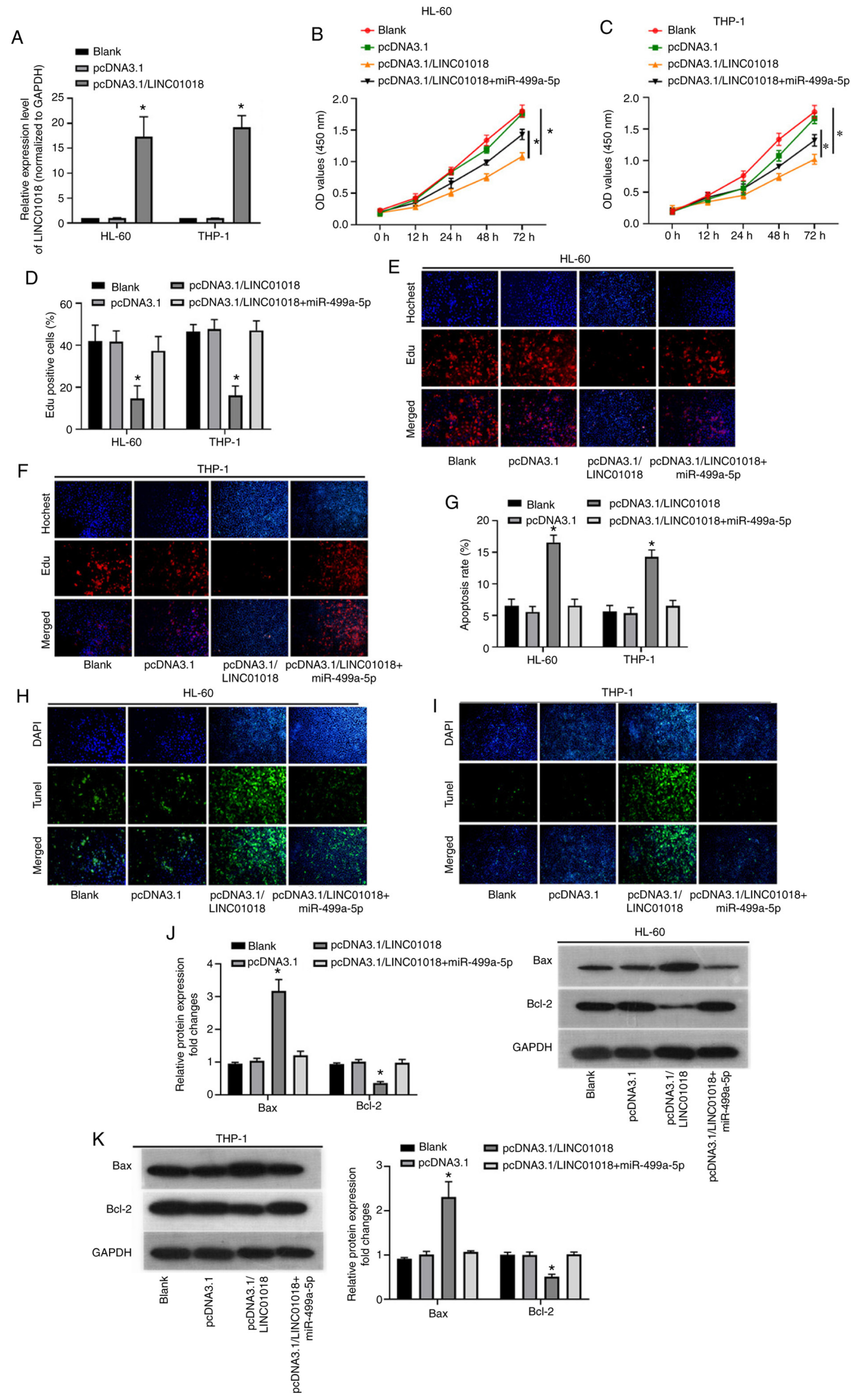

Figure 2. MiR-499a-5p reversed the repressive effects of LINC01018-overexpression on cell growth in AML cell lines. (A) The expression level of LINC01018 was assessed using reverse transcription-quantitative polymerase chain reaction in HL-60 and THP-1 cell lines following transfection with the LINC01018 plasmid. (B and C) Cell Counting kit- 8 assay was performed to measure the proliferation activity of HL-60 and THP-1 cells following transfection of LINC01018 alone or LINC01018 plus miR-499a-5p. (D-F) Cell proliferation was measured following transfection of LINC01018 alone or LINC01018 plus miR-499a-5p by EdU staining. The proliferation ability value is marked in red. (G-I) TUNEL assay was performed to analyze the apoptosis rate following transfection of LINC01018 alone or LINC01018 plus miR-499a-5p in HL-60 and THP-1 cell lines. (J and K) The effects of transfection of LINC01018 alone or LINC01018 plus miR-499a-5p on Bax and Bcl-2 expression in HL-60 and THP-1 cell lines were determined by western blot assay. "P<0.05. MiR, microRNA; AML, acute myeloid leukemia. 

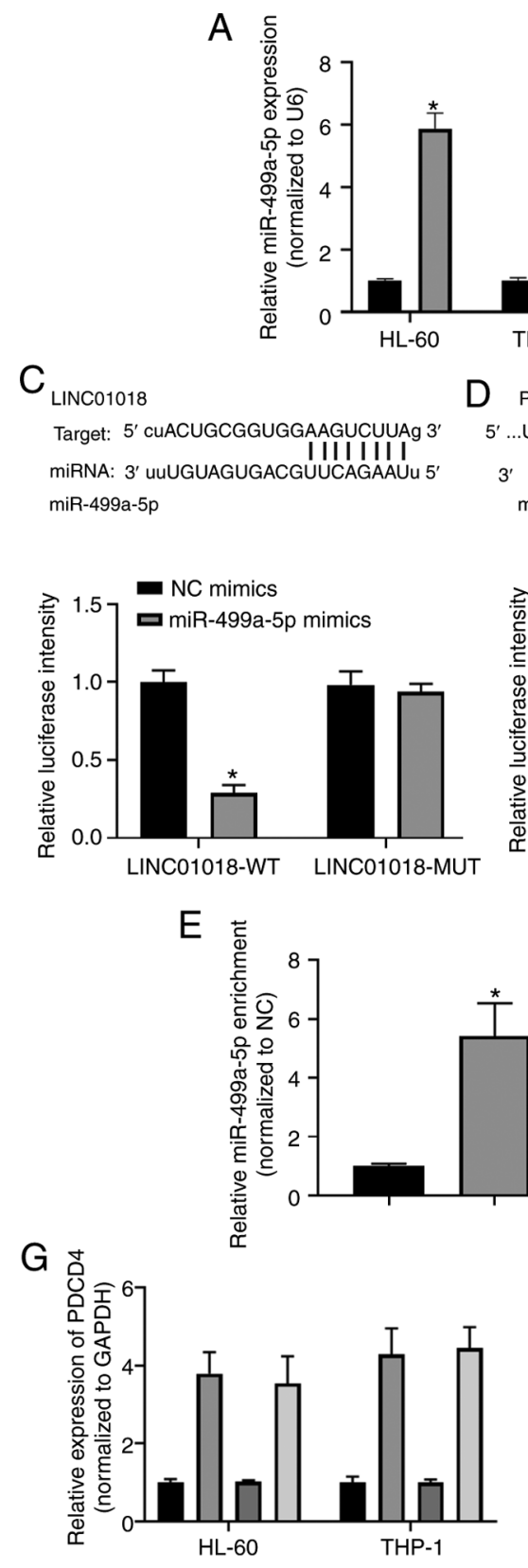

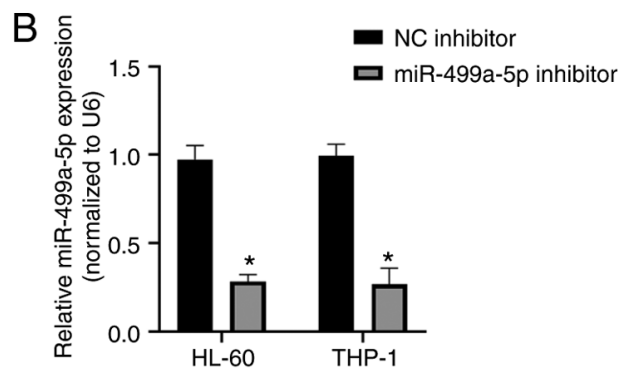
Position 467-473 of PDCD4 3' UTR Position 533-539 of PDCD4 3' UTR 5' ...GAAAGUUAUUUCUUUGUCUUAAA... 5' ...GGAACAUACUGAUUGGUCUUAAA... $3^{\prime}$ UUUGUAGUGACGUUCAGAAUU $\operatorname{miR}-499 a-5 p$
3' UUUGUAGUGACGUUCAGAAUU miR-499a-5p
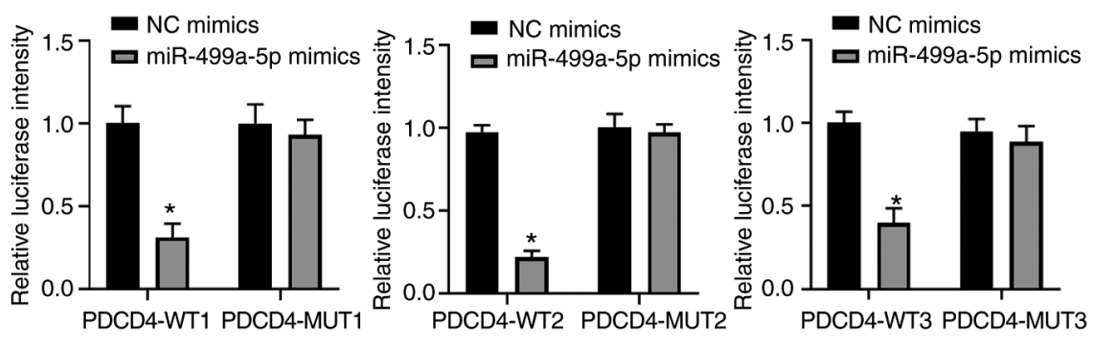

$\mathrm{F}$
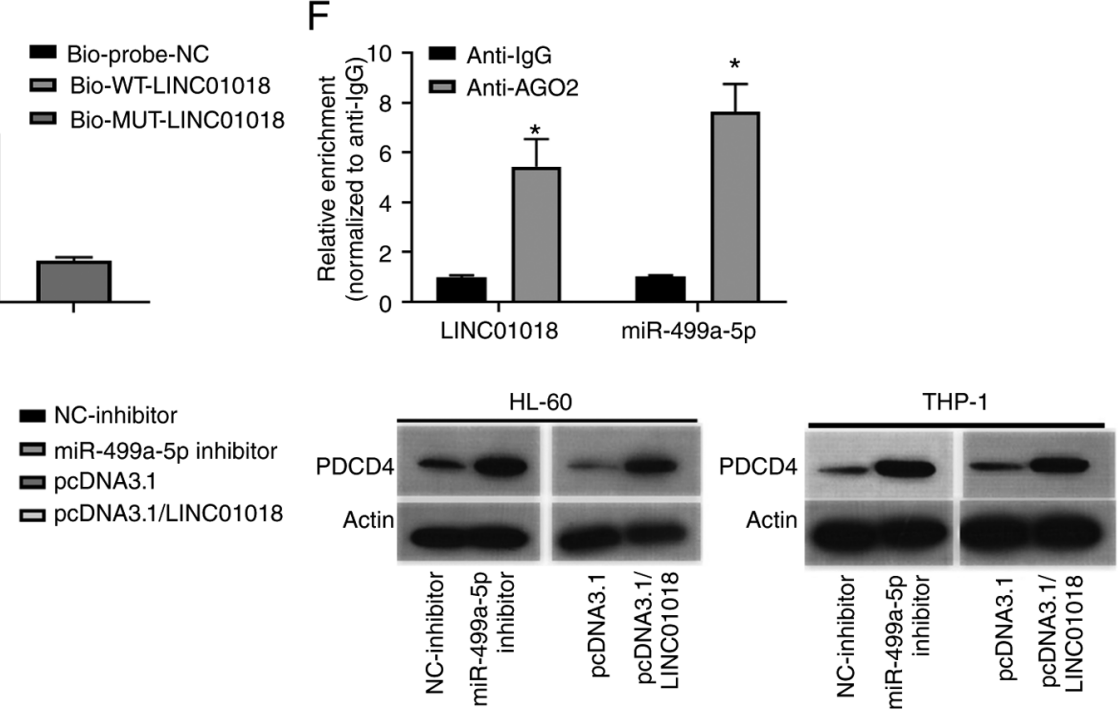

Figure 3. LINC01018 acted as a sponge of miR-499a-5p, and PDCD4 was targeted by miR-499a-5p. (A and B) Reverse transcription-quantitative polymerase chain reaction was performed to detect the miR-499a-5p level in HL-60 and THP-1 cells treated with miR-499a-5p mimics or miR-499a-5p inhibitor. (C) Upper panel, the binding sites of wild-type and mutant LINC01018 and miR-499a-5p. Lower panel, the interaction between LINC01018 and miR-499a-5p was detected using a dual-luciferase reporter assay. (D) Upper panel, three combined sequences of PDCD4 and miR-499a-5p in the 3'-untranslated region binding sites. Lower panel, luciferase reporter experiments were conducted to detect the association between miR-499a-5p and PDCD4. (E and F) RNA pull-down and RIP experiments were performed to confirm the putative target site between LINC01018 and miR-499a-5p. (G) PDCD4 expression in HL-60 and THP-1 cells treated with miR-499a-5p inhibitor or pcDNA3.1/LINC01018 was measured by western blot analysis. *P<0.05. MiR, microRNA; RIP, RNA immunoprecipitation.

that PDCD4 was a target gene of miR-499a-5p, the effects of PDCD4 on miR-499a-5p were investigated. To begin with, a PDCD4-knockdown siRNA was designed and its efficiency was validated (Fig. 4A). Next, western blot analysis of PDCD4 protein expression demonstrated that PDCD4 was significantly increased by transfection of HL-60 and THP-1 cells with a miR-499a-5p inhibitor, while transfection of si-PDCD4 reversed this effect $(\mathrm{P}<0.05$; Fig. $4 \mathrm{~B})$. The EdU proliferation assay was performed to determine the effects of co-transfection of miR-499a-5p inhibitor and si-PDCD4 on cell proliferation.
The results demonstrated that knockdown of miR-499a-5p inhibited the proliferation of HL-60 and THP-1 cells compared with the blank and negative controls, while si-PDCD4 transfection abolished the inhibitory effects of miR-499a-5p on cell proliferation $(\mathrm{P}<0.05$; Fig. $4 \mathrm{C}$ and $\mathrm{D})$. TUNEL assay results demonstrated that the apoptosis rate was upregulated by knockdown of miR-499a-5p in HL-60 and THP-1 cells, while transfection of si-PDCD4 reversed the promotive effects of miR-499a-5p-knockdown on cell apoptosis ( $\mathrm{P}<0.05$; Fig. 4E and F). Furthermore, the expression levels of Bax and Bcl-2 

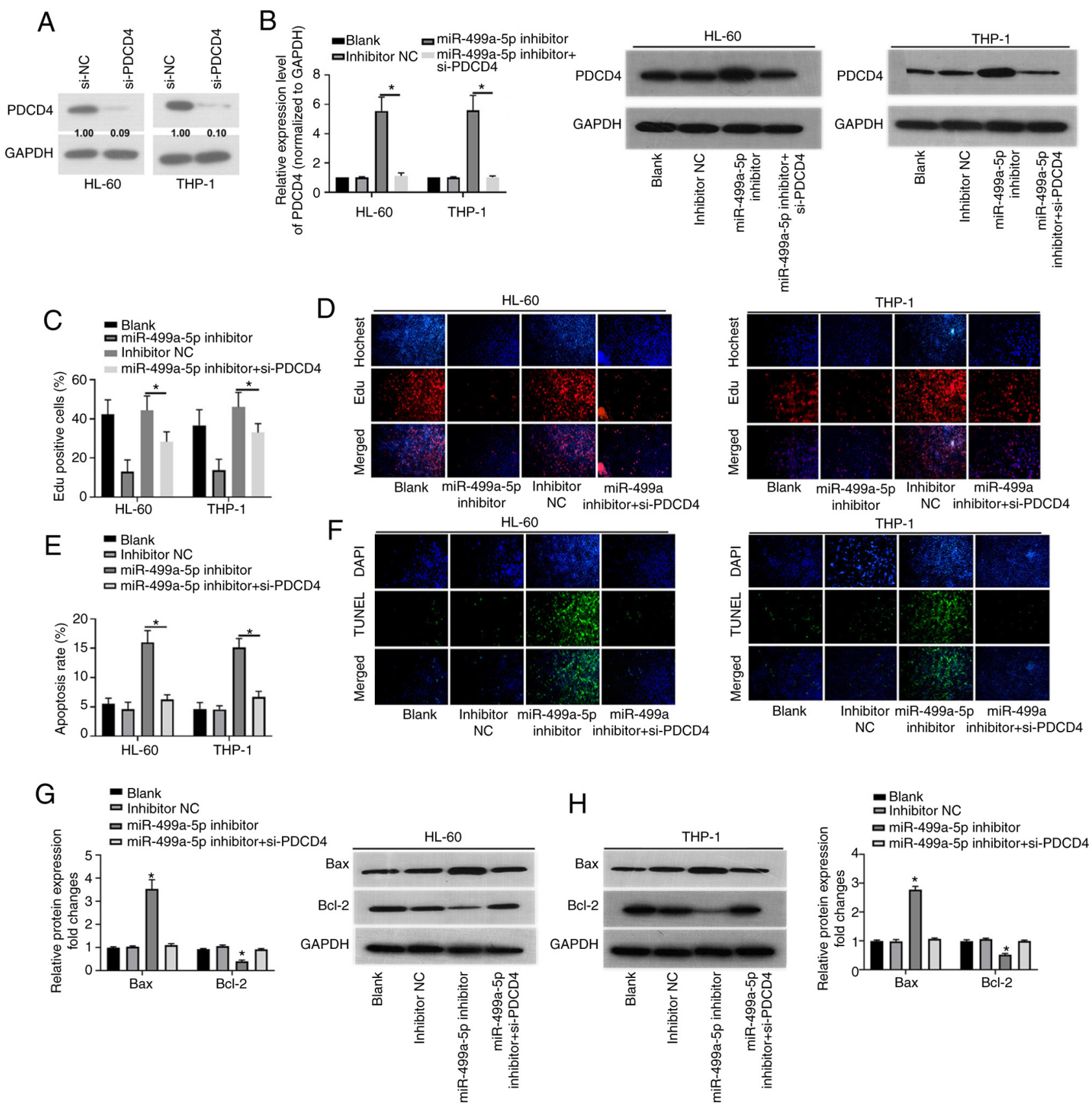

Figure 4. Knockdown of PDCD4 abrogated the effects of miR-499a-5p inhibitor on acute myeloid leukemia cell lines. (A) PDCD4 expression in HL-60 and THP-1 cells treated with si-PDCD4 or siRNA control was detected by western blotting. (B) PDCD4 expression in HL-60 and THP-1 cells treated with miR-499a-5p or miR-499a-5p plus si-PDCD4 was detected by western blotting. (C and D) EdU assay was used to detect cell proliferation after transfection of miR-499a-5p inhibitor alone or miR-499a-5p inhibitor plus si-PDCD4 in HL-60 and THP-1 cells. (E and F) Apoptosis following transfection of miR-499a-5p inhibitor alone or miR-499a-5p inhibitor plus si-PDCD4 was analyzed using TUNEL assay in HL-60 and THP-1 cells. (G and H) Following transfection of miR-499a-5p inhibitor alone or miR-499a-5p plus si-PDCD4, HL-60 and THP-1 cells were subjected to Bax and Bcl-2 expression detection using western blot assay. " $\mathrm{P}<0.05$. siRNA, small interfering RNA; miR, microRNA.

were increased by miR-499a-5p inhibitor transfection, while co-transfection of miR-499a-5p inhibitor and si-PDCD4 abrogated this effect $(\mathrm{P}<0.05$; Fig. $4 \mathrm{G}$ and $\mathrm{H})$. These data demonstrated that the miR-499a-5p inhibitor suppressed the proliferation of HL-60 and THP-1 cells but promoted cell apoptosis, which was accomplished through regulation of PDCD4.

PDCD4 is downregulated in AML. To investigate the correlation between PDCD4 and LINC01018 or miR-499a-5p, the expression level of PDCD4 was measured by RT-qPCR. The results demonstrated that PDCD4 expression was downregulated in HL-60 and THP-1 cells $(\mathrm{P}<0.05$; Fig. 5A) and AML tissues $(\mathrm{P}<0.05$; Fig. $5 \mathrm{~B})$, compared with HS cells and healthy controls. In addition, the results in Fig. 5C demonstrated a positive correlation between PDCD4 and LINC01018 expression levels in patients with AML $(r=0.5098 ; \mathrm{P}=0.0008)$. By contrast, PDCD4 and miR-499a-5p were negatively correlated in patients with AML ( $\mathrm{r}=-0.3342 ; \mathrm{P}=0.0296$; Fig. 5D). In summary, these results suggested that LINC01018 
A

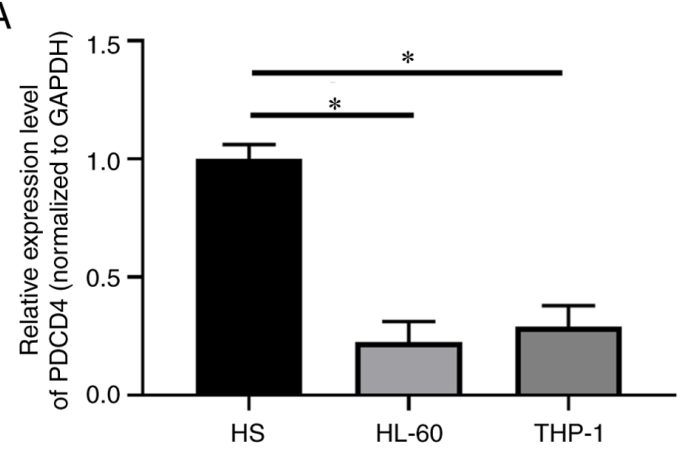

C

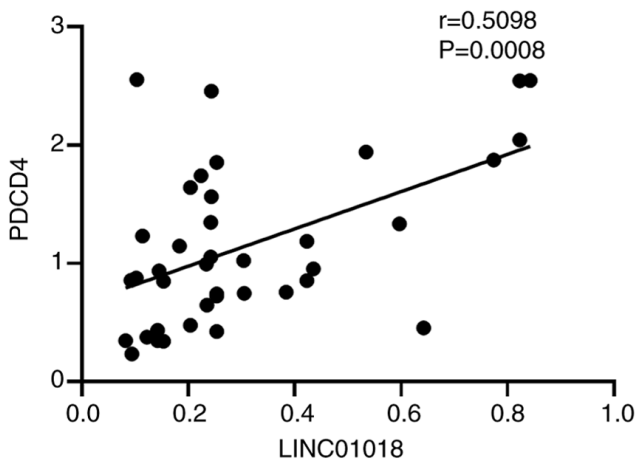

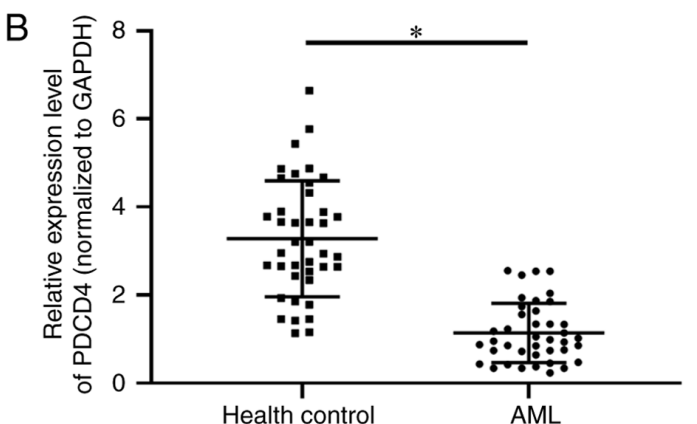

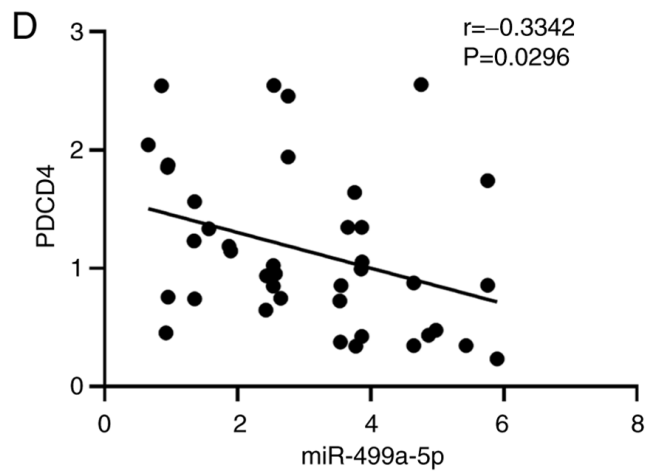

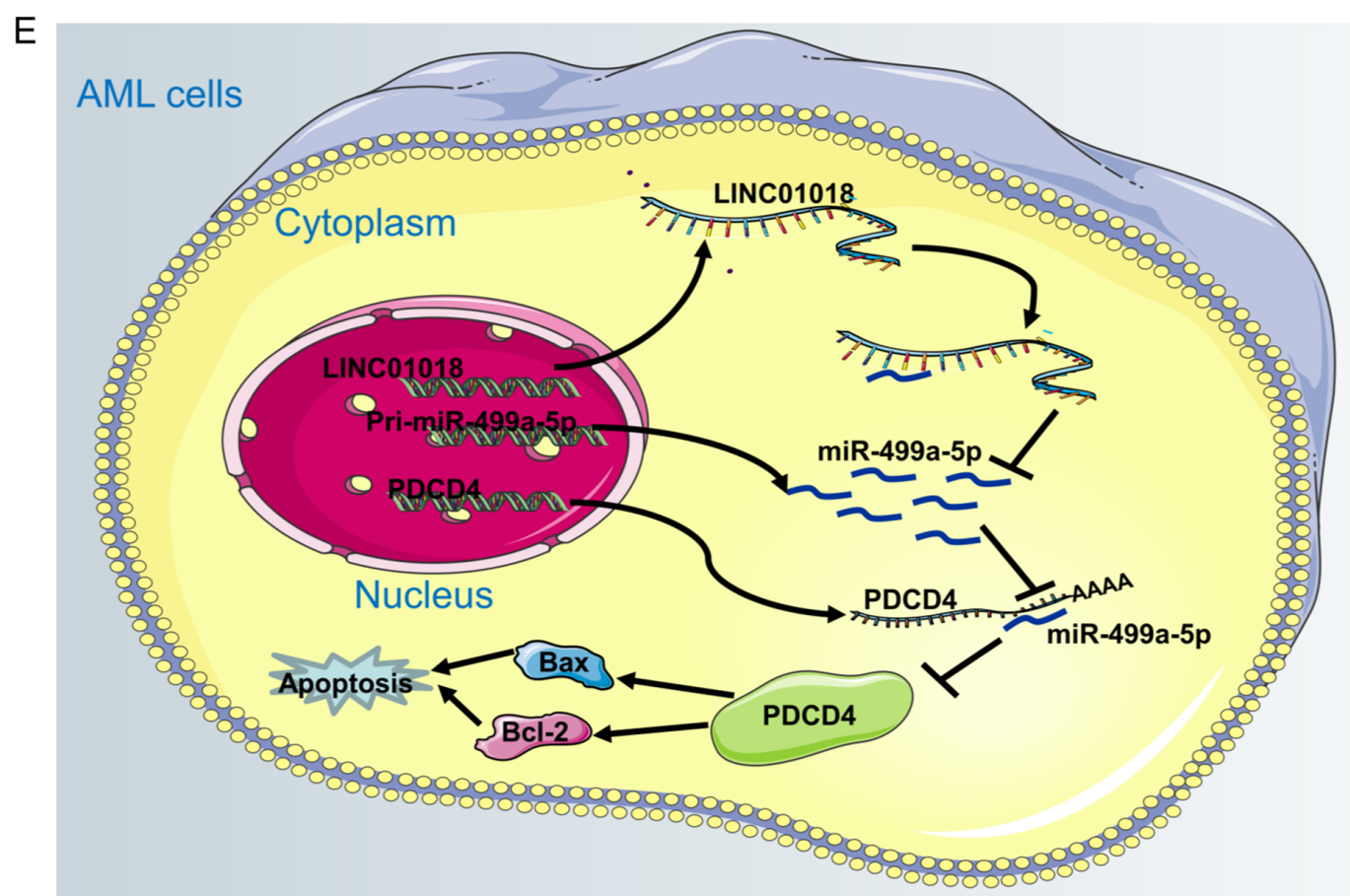

Figure 5. PDCD4 was downregulated in AML. (A) PDCD4 expression in HL-60 and THP-1 cells was estimated via RT-qPCR. HS cells were used as a control. (B) PDCD4 expression in AML and healthy tissues was assessed using RT-qPCR. (C and D) Correlation between the levels of miR-499a-5p or LINC01018 and PDCD4 was measured in 40 AML tissues. (E) Interaction mechanisms of LINC01018, miR-499a-5p and PDCD4 in AML cells. *P<0.05. AML, acute myeloid leukemia; RT-qPCR, reverse transcription-quantitative polymerase chain reaction; miR, microRNA.

contributed toward AML cell growth by modulating PDCD4 through suppression of miR-499a-5p (Fig. 5E).

\section{Discussion}

Increasing evidence has suggested that several lncRNAs are involved in multiple cell processes in AML. Wang et al (34) reported that the IncRNA, CRNDE, which inhibits the apoptosis and promotes the proliferation of U937 cells, acted as a molecular marker for the treatment of AML. Linc-223 was reported to be decreased in AML cells and regulated their proliferation and differentiation (34). A novel axis of LINC01018/miR-182-5p/FOXO1 was identified in hepatocellular carcinoma, and the antitumor effect of LINC01018 was 
verified in vivo (35). However, no previous studies have identified the mechanism of LINC01018 in AML. The present study confirmed the decreased expression of LINC01018 in AML tissues and cell lines, and that LINC01018-overexpression inhibited cell proliferation and promoted apoptosis in AML cells. Therefore, the antitumor effect of LINC01018 in AML has also been substantiated.

At present, numerous studies have confirmed that miRNAs serve key roles in the initiation and progression of AML. For example, miR-34a exhibited lower expression and was identified as a tumor suppressor promoting apoptosis in AML cell lines (36). A study proved that miR-125b induces myeloid and B-cell leukemia by suppressing IRF4 through different mechanisms (37). MiR-135a was revealed to be downregulated in AML cells, and its overexpression inhibited proliferation and the cell cycle and promoted cellular apoptosis via HOXA10 (38). MiR-182-5p promotes cell proliferation in AML cell lines and patient blood samples and reverses cisplatin resistance (39). The molecular mechanism of miR-499a-5p has previously been demonstrated in certain cancer studies (24,40-42). MiR-499a-5p exhibits high expression and carcinogenic effects through the TOR pathway in highly metastatic lung cancer exosomes (22). MiR-499a-5p acts as a non-invasive biomarker and may increase the diagnostic sensitivity of pancreatic cancer when combined with CA199 (24). Nevertheless, the mechanisms of miR-499a-5p in AML pathogenesis remain largely unknown. The results of the present study suggested that miR-499a-5p was upregulated in AML tissues and cell lines, knockdown of miR-499a-5p suppressed cell proliferation and induced cell apoptosis, and miR-499a-5p was sponged by LINC01018. A negative correlation was identified between LINC01018 and miR-499a-5p.

PDCD4 may interfere with the translation process by directly binding with target mRNA. Multiple targets of PDCD4 are involved in cell survival, proliferation and invasion. Identifying more translational targets of PDCD4 will provide insight into how PDCD4 inhibits tumorigenesis. For example, in CML primary $\mathrm{CD} 4^{+}$cells and AML cell models, including MOLM13 and MV4.11, the phospho-STAT5-miR21-PDCD4 pathway is active (43). Several studies have demonstrated that PDCD4, as a target gene of miRNAs, serves an important role in AML (44-48). These findings are consistent with the results of the present study. In the present study the expression levels of PDCD4 in AML tissues and cell lines were measured, and the results revealed high expression. Next, bioinformatics analysis and the dual-luciferase reporter assay were used to determine that PDCD4 was the target gene of miR-499a-5p, and knockdown of PDCD4 could reverse the effects of the miR-499a-5p inhibitor on AML progression.

In summary, the results of the present study suggested that the low level of LINC01018 is associated with AML pathogenesis by inhibiting AML cell proliferation and promoting apoptosis, which is mediated via knockdown of miR-499a-5p and regulation of PDCD4 expression. These observations provide a feasible theoretical basis for the treatment of AML.

\section{Acknowledgements}

Not applicable.

\section{Funding}

The present study was supported by the National Natural Science Foundation of China (grant no. 81600129) and the Science and Technology Project of Hangzhou (grant no. 2016Z01).

\section{Availability of data and materials}

The datasets used during the present study are available from the corresponding author upon reasonable request.

\section{Authors' contributions}

HZ and QX designed the experiments. HZ, QX, PS and XJ performed the experiments. HZ collected the data. QX analyzed the data. HZ and QX drafted the initial manuscript. All authors have read and approved the final manuscript.

\section{Ethics approval and consent to participate}

The present study was approved by the Ethics Committee of Affiliated Hangzhou First People's Hospital (Hangzhou, China; approval no. 146-01). Written informed consent was provided by all patients prior to the study start.

\section{Patient consent for publication}

Not applicable.

\section{Competing interests}

The authors declare that they have no competing interests.

\section{References}

1. De Kouchkovsky I and Abdul-Hay M: 'Acute myeloid leukemia: A comprehensive review and 2016 update'. Blood Cancer J 6: e441, 2016.

2. Shah A, Andersson TM, Rachet B, Björkholm M and Lambert PC: Survival and cure of acute myeloid leukaemia in England, 1971-2006: A population-based study. Br J Haematol 162: 509-516, 2013.

3. Davila J, Slotkin E and Renaud T: Relapsed and refractory pediatric acute myeloid leukemia: Current and emerging treatments. Paediatr Drugs 16: 151-168, 2014.

4. Meyers J, Yu Y, Kaye JA and Davis KL: Medicare fee-for-service enrollees with primary acute myeloid leukemia: An analysis of treatment patterns, survival, and healthcare resource utilization and costs. Appl Health Econ Health Policy 11: 275-286, 2013.

5. Shi Z, Tiwari AK, Patel AS, Fu LW and Chen ZS: Roles of sildenafil in enhancing drug sensitivity in cancer. Cancer Res 71: 3735-3738, 2011.

6. Yang JX, Rastetter RH and Wilhelm D: Non-coding RNAs: An introduction. Adv Exp Med Biol 886: 13-32, 2016.

7. Dong X, Chen K, Cuevas-Diaz Duran R, You Y, Sloan SA, Zhang Y, Zong S, Cao Q, Barres BA and Wu JQ: Comprehensive identification of long non-coding RNAs in purified cell types from the brain reveals functional LncRNA in OPC fate determination. PLoS Genet 11: e1005669, 2015.

8. Wrang X, Chen H, Bai J and He A: MicroRNA: An important regulator in acute myeloid leukemia. Cell Biol Int 41: 936-945, 2017.

9. Lei L, Xia S, Liu D, Li X, Feng J, Zhu Y, Hu J, Xia L, Guo L, Chen F, et al: Genome-wide characterization of lncRNAs in acute myeloid leukemia. Brief Bioinform 19: 627-635, 2018.

10. Jarroux J, Morillon A and Pinskaya M: History, discovery, and classification of lncRNAs. Adv Exp Med Biol 1008: 1-46, 2017.

11. Puvvula PK: LncRNAs regulatory networks in cellular senescence. Int J Mol Sci 20: 2615, 2019. 
12. Maruyama R and Suzuki H: Long noncoding RNA involvement in cancer. BMB Rep 45: 604-611, 2012.

13. Yang Z, Zhou L, Wu LM, Lai MC, Xie HY, Zhang F and Zheng SS Overexpression of long non-coding RNA HOTAIR predicts tumor recurrence in hepatocellular carcinoma patients following liver transplantation. Ann Surg Oncol 18: 1243-1250, 2011.

14. Kogo R, Shimamura T, Mimori K, Kawahara K, Imoto S, Sudo T, Tanaka F, Shibata K, Suzuki A, Komune S, et al: Long noncoding RNA HOTAIR regulates polycomb-dependent chromatin modification and is associated with poor prognosis in colorectal cancers. Cancer Res 71: 6320-6326, 2011.

15. Kim K, Jutooru I, Chadalapaka G, Johnson G, Frank J, Burghardt R, Kim S and Safe S: HOTAIR is a negative prognostic factor and exhibits pro-oncogenic activity in pancreatic cancer. Oncogene 32: 1616-1625, 2013.

16. Ji P, Diederichs S, Wang W, Böing S, Metzger R, Schneider PM Tidow N, Brandt B, Buerger H, Bulk E, et al: MALAT-1, a novel noncoding RNA, and thymosin beta4 predict metastasis and survival in early-stage non-small cell lung cancer. Oncogene 22 8031-8041, 2003

17. Miao Y, Sui J, Xu SY, Liang GY, Pu YP and Yin $\mathrm{LH}$ Comprehensive analysis of a novel four-lncRNA signature as a prognostic biomarker for human gastric cancer. Oncotarget 8: 75007-75024, 2017.

18. Ruan X, Li P, Chen Y, Shi Y, Pirooznia M, Seifuddin F, Suemizu H, Ohnishi Y, Yoneda N, Nishiwaki M, et al: In vivo functional analysis of non-conserved human lncRNAs associated with cardiometabolic traits. Nat Commun 11: 45, 2020.

19. Shang H, Sun L, Braun T, Si Q and Tong J: Association between miR-124 rs531564 and miR-100 rs1834306 polymorphisms and cervical cancer: A meta-analysis. Eur J Gynaecol Oncol 40: 925-931, 2019.

20. Song SJ, Ito K, Ala U, Kats L, Webster K, Sun SM Manova-Todorova K, Teruya-Feldstein J, Avigan DE, Delwel R and Pandolfi PP: The oncogenic microRNA miR-22 targets the TET2 tumor suppressor to promote hematopoietic stem cell self-renewal and transformation. Cell Stem Cell 13: 87-101, 2013.

21. Bousquet M, Quelen C, Rosati R, Mansat-De Mas V, La Starza R, Bastard C, Lippert E, Talmant P, Lafage-Pochitaloff M, Leroux D, et al: Myeloid cell differentiation arrest by miR-125b-1 in myelodysplastic syndrome and acute myeloid leukemia with the t(2;11)(p21;q23) translocation. J Exp Med 205: 2499-2506, 2008.

22. He S, Li Z, Yu Y, Zeng Q, Cheng Y, Ji W, Xia W and Lu S: Exosomal miR-499a-5p promotes cell proliferation, migration and EMT via mTOR signaling pathway in lung adenocarcinoma. Exp Cell Res 379: 203-213, 2019.

23. Hou YY, Lee JH, Chen HC, Yang CM, Huang SJ, Liou HH, Chi CC, Tsai KW and Ger LP: The association between miR-499a polymorphism and oral squamous cell carcinoma progression. Oral Dis 21: 195-206, 2015

24. Shi Q, Feng K, Xia L, Wang C and Zhu J: Combined use of Serum miR-499a-5p and CA199 increases the diagnostic sensitivity of pancreatic cancer. Clin Lab 65, 2019.

25. Elmore S: Apoptosis: A review of programmed cell death. Toxicol Pathol 35: 495-516, 2007.

26. Manabe A, Coustan-Smith E, Kumagai M, Behm FG, Raimondi SC, Pui CH and Campana D: Interleukin-4 induces programmed cell death (apoptosis) in cases of high-risk acute lymphoblastic leukemia. Blood 83: 1731-1737, 1994.

27. Long J, Yin Y, Guo H, Li S, Sun Y, Zeng C and Zhu W: The mechanisms and clinical significance of PDCD4 in colorectal cancer. Gene 680: 59-64, 2019.

28. Wang Q and Yang HS: The role of Pded4 in tumour suppression and protein translation. Biol Cell: May 28, 2018 (Epub ahead of print).

29. Zhou H and Huang S: Role of mTOR signaling in tumor cell motility, invasion and metastasis. Curr Protein Pept Sci 12: 30-42, 2011.

30. Fehler O, Singh P, Haas A, Ulrich D, Müller JP, Ohnheiser J and Klempnauer KH: An evolutionarily conserved interaction of tumor suppressor protein Pdcd4 with the poly(A)-binding protein contributes to translation suppression by Pdcd4. Nucleic Acids Res 42: 11107-11118, 2014.
31. Liwak U, Thakor N, Jordan LE, Roy R, Lewis SM, Pardo OE, Seckl M and Holcik M: Tumor suppressor PDCD4 represses internal ribosome entry site-mediated translation of antiapoptotic proteins and is regulated by S6 kinase 2. Mol Cell Biol 32: 1818-1829, 2012.

32. Wedeken L, Singh P and Klempnauer KH: Tumor suppressor protein Pdcd4 inhibits translation of p53 mRNA. J Biol Chem 286: 42855-42862, 2011.

33. Livak KJ and Schmittgen TD: Analysis of relative gene expression data using real-time quantitative PCR and the 2(-Delta Delta C(T)) method. Methods 25: 402-408, 2001

34. Wang Y, Zhou Q and Ma JJ: High expression of lnc-CRNDE presents as a biomarker for acute myeloid leukemia and promotes the malignant progression in acute myeloid leukemia cell line U937. Eur Rev Med Pharmacol Sci 22: 763-770, 2018.

35. Wang S, Xu M, Sun Z, Yu X, Deng Y and Chang H: LINC01018 confers a novel tumor suppressor role in hepatocellular carcinoma through sponging microRNA-182-5p. Am J Physiol Gastrointest Liver Physiol 317: G116-G126, 2019.

36. Liu L, Ren W and Chen K: MiR-34a promotes apoptosis and inhibits autophagy by targeting HMGB1 in acute myeloid leukemia cells. Cell Physiol Biochem 41: 1981-1992, 2017.

37. So AY, Sookram R, Chaudhuri AA, Minisandram A, Cheng D, Xie C, Lim EL, Flores YG, Jiang S, Kim JT, et al: Dual mechanisms by which miR-125b represses IRF4 to induce myeloid and B-cell leukemias. Blood 124: 1502-1512, 2014.

38. Xu H and Wen Q: Downregulation of miR135a predicts poor prognosis in acute myeloid leukemia and regulates leukemia progression via modulating HOXA10 expression. Mol Med Rep 18: 1134-1140, 2018.

39. Zhang S, Zhang Q, Shi G and Yin J: MiR-182-5p regulates BCL2L12 and BCL2 expression in acute myeloid leukemia as a potential therapeutic target. Biomed Pharmacother 97: 1189-1194, 2018

40. Gu X, Dong M, Liu Z, Yang J and Shi Y: MiR-499a-5p inhibits proliferation, invasion, migration, and epithelial-mesenchymal transition, and enhances radiosensitivity of cervical cancer cells via targeting eIF4E. Onco Targets Ther 13: 2913-2924, 2020.

41. Yang Z, Li C, Fan XY and Liu LJ: Circular RNA circ_0079593 promotes glioma development through regulating KPNA 2 expression by sponging miR-499a-5p. Eur Rev Med Pharmacol Sci 24: 1288-1301, 2020.

42. Zhao L, Jiang P, Zheng H, Chen P and Yang M: Downregulation of miR-499a-5p predicts a poor prognosis of patients with non-small cell lung cancer and restrains the tumorigenesis by targeting fibroblast growth factor 9. Technol Cancer Res Treat 19: $1533033820957001,2020$.

43. Espadinha AS, Prouzet-Mauléon V, Claverol S, Lagarde V, Bonneu M, Mahon FX and Cardinaud B: A tyrosine kinase-STAT5-miR21-PDCD4 regulatory axis in chronic and acute myeloid leukemia cells. Oncotarget 8: 76174-76188, 2017.

44. Gu J, Zhu X, Li Y, Dong D, Yao J, Lin C, Huang K, Hu H and Fei J: miRNA-21 regulates arsenic-induced anti-leukemia activity in myelogenous cell lines. Med Oncol 28: 211-218, 2011.

45. Ozpolat B, Akar U, Steiner M, Zorrilla-Calancha I, Tirado-Gomez M, Colburn N, Danilenko M, Kornblau S and Berestein GL: Programmed cell death-4 tumor suppressor protein contributes to retinoic acid-induced terminal granulocytic differentiation of human myeloid leukemia cells. Mol Cancer Res 5: 95-108, 2007.

46. Riccioni R, Lulli V, Castelli G, Biffoni M, Tiberio R, Pelosi E, Lo-Coco F and Testa U: miR-21 is overexpressed in NPM1-mutant acute myeloid leukemias. Leuk Res 39: 221-228, 2015.

47. Simmons HM, Ruis BL, Kapoor M, Hudacek AW and Conklin KF: Identification of NOM1, a nucleolar,eIF4A binding protein encoded within the chromosome 7q36 breakpoint region targeted in cases of pediatric acute myeloid leukemia. Gene 347: 137-145, 2005

48. Wang JJ, Wang ZY, Chen R, Xiong J, Yao YL, Wu JH and Li GX Macrophage-secreted exosomes delivering miRNA-21 inhibitor can regulate BGC-823 cell proliferation. Asian Pac J Cancer Prev 16: 4203-4209, 2015.

This work is licensed under a Creative Commons Attribution-NonCommercial-NoDerivatives 4.0 International (CC BY-NC-ND 4.0) License. 